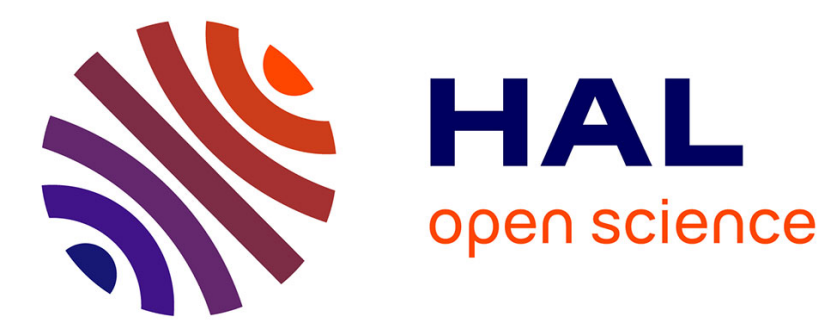

\title{
Equilibrium shape of nickel crystal
}

Ju-Seop Hong, Wook Jo, Kyung-Jun Ko, Nong Moon Hwang, Doh-Yeon Kim

\section{To cite this version:}

Ju-Seop Hong, Wook Jo, Kyung-Jun Ko, Nong Moon Hwang, Doh-Yeon Kim. Equilibrium shape of nickel crystal. Philosophical Magazine, 2009, 89 (32), pp.2989-2999. 10.1080/14786430903164598. hal-00526844

\section{HAL Id: hal-00526844 \\ https://hal.science/hal-00526844}

Submitted on 16 Oct 2010

HAL is a multi-disciplinary open access archive for the deposit and dissemination of scientific research documents, whether they are published or not. The documents may come from teaching and research institutions in France or abroad, or from public or private research centers.
L'archive ouverte pluridisciplinaire HAL, est destinée au dépôt et à la diffusion de documents scientifiques de niveau recherche, publiés ou non, émanant des établissements d'enseignement et de recherche français ou étrangers, des laboratoires publics ou privés. 


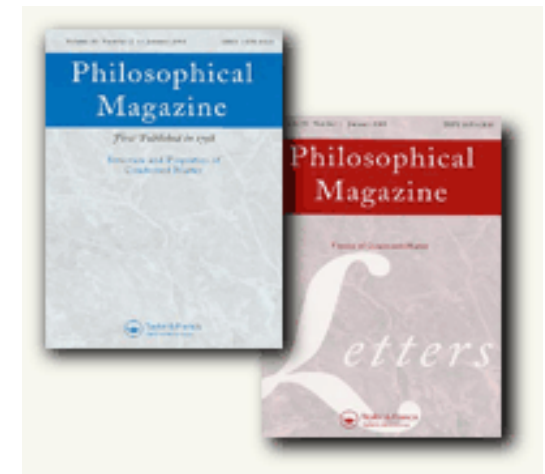

\section{Equilibrium shape of nickel crystal}

\begin{tabular}{|c|c|}
\hline Journal: & Philosophical Magazine \& Philosophical Magazine Letters \\
\hline Manuscript ID: & TPHM-09-May-0187.R1 \\
\hline Journal Selection: & Philosophical Magazine \\
\hline $\begin{array}{r}\text { Date Submitted by the } \\
\text { Author: }\end{array}$ & 23-Jun-2009 \\
\hline Complete List of Authors: & $\begin{array}{l}\text { Hong, Ju-Seop; Seoul National University, Department of Materials } \\
\text { Science \& Engineering } \\
\text { Jo, Wook; TEchnische Universität Darmstadt, Institute of Materials } \\
\text { Science } \\
\text { Ko, Kyung-Jun; Seoul National University, Department of Materials } \\
\text { Science \& Engineering } \\
\text { Hwang, Nong Moon; Seoul National University, Materials Science } \\
\text { and Engineering } \\
\text { Kim, Doh-Yeon; Seoul National University, Department of Materials } \\
\text { Science \& Engineering }\end{array}$ \\
\hline Keywords: & equilibrium shape, nickel \\
\hline Keywords (user supplied): & surface energy anisotropy \\
\hline
\end{tabular}

\section{今 scholaroNE" \\ Manuscript Central}




\section{Equilibrium shape of nickel crystal}

Ju-Seop Hong, Wook Jo\#, Kyung-Jun Ko, Nong Moon Hwang, and

Doh-Yeon Kim

Department of Materials Science \& Engineering, Seoul National University, Kwanak-gu, Seoul 151-744, Korea

Complete list of correspondence:

: Ju-Seop Hong

tel:+82-2-880-9152, fax:+82-2-884-1413, email: kidhong7@ @nu.ac.kr

Wook Jo

tel:+49-6151-16-6316, fax:+49-6151-16-6314, email: jo@ ceramics.tu-darmstadt.de

Current address: Institute of Materials Science, Technische Universität Darmstadt, Darmstadt,

Germany.

: Kyung-Jun Ko

tel:+82-2-880-9152, fax:+82-2-884-1413, email: kko04@ snu.ac.kr

Nong-Moon Hwang

tel:+82-2-880-8922, fax:+82-2-883-8197, email: nmhwang@ @snu.ac.kr

Doh-Yeon Kim

tel:+82-2-880-7160, fax:+82-2-884-1413, email: dykim@snu.ac.kr

\#All correspondence should be addressed to WJ (jo@ @ceramics.tu-darmstadt.de) 


\section{Abstract}

The equilibrium shape of pure nickel and the effect of carbon on the changes in the equilibrium shape at $1200^{\circ} \mathrm{C}$ were investigated. A statistical observation over the size-dependent, time-dependent, and carbon-induced morphological evolution of crystallites suggested that the equilibrium crystal shape (ECS) of pure nickel is a polyhedron consisting of $\{111\},\{100\},\{110\}$, and $\{210\}$ surfaces. However, crystals with an extensive portion of $\{320\}$ surfaces were also frequently observed. The appearance of $\{320\}$ surfaces were interpreted as kinetically-stabilized metastable surfaces which survived during the thermal equilibrating process possibly due to a high nucleation energy barrier for the removal of the surfaces. On the other hand, the ECS of pure nickel was observed to change drastically into a spherical shape with the facets of $\{111\},\{100\},\{110\}$, and $\{210\}$ without exception under a carburized atmosphere, which indicates that carbon not only facilitates the surface diffusion by which energetically more stable surfaces can be easily developed but also decreases the surface energy anisotropy. Together with X-ray photoelectron spectroscopy studies, it was proposed that the carbon-induced changes in the ECS are possibly due to a solid solution effect which could lead to a reduction in the binding energy among atoms in the bulk as well as on the surfaces.

Keywords: equilibrium crystal shape; surface energy anisotropy; nickel (Ni) 


\section{Introduction}

Nickel has been one of the materials of interest both in academic societies and in industries due to its excellent mechanical and thermal properties. During the last decade, special attention has been paid to the processing related aspects because nickel is not only a key element for superalloys [1], but also an alternative electrode material for multilayer ceramic capacitors (MLCCs) [2]. Especially in the case of MLCC applications, obtaining a homogeneous microstructure with a uniform grain size distribution is one of the key issues in guaranteeing the consistency and reliability of the expected functional properties. It is known that pure nickel often exhibits abnormal grain growth (AGG) behavior during sintering or annealing [3, 4], which imposes a great challenge to the industrial side. As well, the presence of impurities such as carbon is known to often result in a homogeneous microstructure [4]. It follows that the understanding of the mechanism which can clarify the microstructure evolution of the nickel system would be a very important subject.

Doping foreign elements is commonly known to be highly effective in controlling microstructures of materials. In fact, changes in ECS due to the incorporation of foreign elements have been frequently observed and reported in various systems including ceramics and metals [5], even though a decisive theoretical understanding is still lacking. One of the two most frequently-cited mechanisms for the microstructure 
The other mechanism states that the coarsening behavior of polycrystalline materials is highly susceptible to changes in the anisotropy of the surface energies [9-11]. This mechanism predicts that the higher the anisotropy of the surface energies of a crystal, the higher the probability that AGG can take place during the sintering of polycrystalline materials.

Recently, Lee et al. [4] reported that the addition of a small amount of carbon is highly effective in suppressing the AGG of nickel. They proposed that the suppressed AGG in the presence of carbon could be due to a decrease in the anisotropy of the interfacial energies from the fact that grain boundary faceting is only observed in pure nickel systems. In the meantime, Randle and Horton [3] reported that the presence of impurities during the sintering of nickel tends to suppress the AGG. It is interesting to note that the Zener dragging effect as a working mechanism specifically in the case of the effect of carbon on the microstructure evolution of nickel does not seem to be relevant, because the microstructure homogenization effect evidently takes place even in systems where the doping level of carbon is fairly below the solubility limit [12]. Note that when the doping level is below the solubility limit, neither a solute atom nor a secondary phase, which could retard the migration of the grain boundaries, is expected. Therefore, at least the effect of carbon on homogenizing the microstructure of nickel could be attributed to a reduction in the anisotropy of the surface energies. 
The best way to monitor the changes in the surface energy anisotropy is to compare the ECS of each system.

In principle, there are two ways to experimentally induce the ECS of a crystal. One that is the most straightforward, though it is only qualitatively acceptable [13-15] is to monitor the shape of isolated crystals in a closed system at a specific temperature of interest $[16,17]$. When a consensus on the shape of the majority of crystals is reached, the shape can be approximated as the ECS. The other is to analyze a so-called negative shape of crystals from nano-sized annealed cavities[18, 19]. From the experimental point of view, the latter method is easier and more reliable since the size of cavities can be as small as a few nanometers, which allows the shape to reach the equilibrium state relatively easier $[13,20]$. However, a couple of disadvantages of this method is that it only provides two-dimensional information, which is not quite practical in analyzing the ECS with a low symmetry, and the applicability of the technique is usually confined to ceramic systems that are brittle enough to generate crack-induced cavities.

Considering the ductile nature of nickel as an elementary metallic system, we investigated the ECS of both pure and carburized nickel crystals by the former method, i.e. from the positive crystal shape. To induce the isolated crystals with sizes in the micrometer range, we used sapphire single crystals as a substrate on which the 
wettability of nickel is known to be very poor [21]. Although the best way to break up deposited films into isolated single crystals is to heat-treat them above their melting point where the surface tension is operative to form small droplets, all the specimens were merely heat-treated at $1200^{\circ} \mathrm{C}$ due to the significant solubility of aluminum in molten nickel [22]. The comparison of the ECS's suggested that carbon plays an important role in reducing the surface energy anisotropy of the nickel system, though the operating mechanism is not satisfactorily explained from the existing theory that states the changes in the surface energy anisotropy is merely a consequence of impurity adsorption on surfaces. From the analyses on the phenomenological aspects monitored by a scanning electron microscopy (SEM) and X-ray photoelectron spectroscopy (XPS), a possibility was proposed that the changes in the surface energy anisotropy in the presence of impurities may also be induced by a solid-solution. 


\section{Experimental Procedure}

Continuous nickel thick films were deposited on sapphire single crystal substrates of high purity of $99.995 \%(10 \times 10 \times 1 \mathrm{~mm}$, ILJIN Display Co., Ltd, Korea) at room temperature. Since even a small amount of impurities could result in a notable deviation from the real ECS, highly pure nickel $(99.99 \%, 4$ inch dia., 3mm thick, Kojundo Chemical Laboratory Co., Ltd, Japan) was used as a target material, and the sputtering was carried out by a DC magnetron device (VSDS-81A, Vacuum Science Co., Ltd, Korea). To minimize possible contaminants, which could act as ECS modifiers, the base pressure was set to be $10^{-6}$ torr. For the generation of a plasma that facilitates the sputtering process, a continuous and steady flow of argon gas (99.9999\%) was purged into the chamber to make the chamber pressure constantly maintained at $5 \times 10^{-2}$ torr. Under these deposition conditions, nickel films with a fairly uniform thickness of $\sim 300 \mathrm{~nm}$ were obtained as shown in Fig. 1 .

All the deposited specimens were sealed in a highly pure quartz tube in vacuo $\left(10^{-4}\right.$ torr), and then placed in a vacuum furnace (JMVF-HT-3, Jeong Min Industry, Korea). The isolation and thermal equilibration of the deposited nickel films were carried out at $1200^{\circ} \mathrm{C}$ for $100 \mathrm{~h}$ at $6 \times 10^{-3}$ torr. This heat-treatment condition was chosen on the basis of preliminary experiments. Although the heat-treatment was carried out at the 
temperature that is fairly below the melting point, it is seen that nicely dispersed and isolated microcrystallites were obtained from the deposited film. As shown in Fig. 2 (a) and (b), the size distribution and highly uniform contact angle of $\sim 130^{\circ}$ between the nickel crystals and a (0001) oriented sapphire substrate indicates that the formation of nickel microcrystallites of high crystallinity is the consequence of the heterogeneous nucleation and growth process. It is interesting to note that the uniform contact angle between nickel crystals and the substrate is even larger than the known wetting angle between liquid nickel droplet and the sapphire [23]. The size of the isolated nickel crystallites ranged from about 0.5 to $3 \mu \mathrm{m}$, as is clearly visible in Fig. 2 (b). Except the crystals that inherently conceive a twin or low-angle grain boundaries, all the observed crystals showed practically the identical shape, which indicates that they are very close to their equilibrium state. The only indication that the system is still away from the equilibrium shape was a slight deviation in the areal fraction of each surface on the ECS. However, the deviation became less perceptible within the resolution limit of the SEM used (JSM-6330F, JEOL, Japan) when the crystal size was near or below a micrometer.

Some of the specimens were then resealed in a quartz tube under the same condition to induce the ECS of carburized nickel crystals. To introduce carbon into each isolated nickel crystal, a carburizing atmosphere was realized by placing a powder compact, which was made of $4 \mathrm{wt} \%$ nickel and $96 \mathrm{wt} \%$ carbon powder mixture directly next to 
the specimen inside the quartz tube. The use of a carbon-nickel mixture instead of pure carbon as a source of the carburizing atmosphere was to avoid a sudden increase in the vapor pressure of carbon and to maintain as stable a supply of carbon to the specimens during the heat-treatment as possible. These specimens were also annealed at $1200^{\circ} \mathrm{C}$ for $100 \mathrm{~h}$. The morphological aspects of the ECS from each specimen were monitored by SEM and then characterized by Wulffman software [24]. Any change in the bonding features of nickel crystals induced by the incorporation of carbon was evaluated by using an X-ray photoelectron spectroscopy (Kratos, Axis, England) method. 


\section{Results \& discussion}

It was observed that all the induced near-ECS's of the pure nickel system belong to one of the two representative shapes shown in Figs. 3 (a) and (b). Notice that these two shapes are identical except the symmetry of the surface whose normal vector is nearly parallel to the substrate normal. From the rotational symmetries of the surfaces, i.e. the one in Fig. 3 (a) is 6-fold and the other in Fig. 3 (b) is 3-fold, it is evident that the top surface is $\{111\}$ which has the highest symmetry for the face centered cubic (fcc) systems. It is interesting to note that the nickel crystals generated are crystallographically very coherent to the substrate, though the lattice mismatch between the nickel $\{111\}$ surface and sapphire $\{0001\}$ surface is relatively high. This implicitly indicates that there might be a buffer layer between the grown nickel crystals and the substrate, which could be formed by a diffusion of aluminum from the substrate [22]. Considering the symmetry constraints imposed on the fcc crystals, a crystallographic characterization of the observed facets is rather straightforward except the surfaces in contact with the $\{111\}$ surfaces, e.g. 6 surfaces in contact with the top $\{111\}$ surface. The determination of the surfaces will be further discussed later in detail. A statistical observation on all the crystals from the smallest to the largest revealed that $\{100\}$ surfaces with a 4-fold rotational symmetry and $\{110\}$ surfaces with a 2-fold rotational symmetry are also a part of the ECS. 
On the other hand, the shape of all the crystals formed under the carburizing atmosphere showed a drastic difference from that of the pure crystals. When observed at low magnification, the shape of each crystal is practically a sphere regardless of the crystallite size. This means that the role of carbon in nickel systems is obviously a surface energy anisotropy reducer. However, a close look on the individual crystals reveals that the induced spherical crystallites are intersected by several discrete facets as exemplarily shown in Fig. 4 (a) and (b). Similar to the case of pure nickel crystals, the facet on the top is easy to be identified as a $\{111\}$ surface, since the $\{0001\}$ oriented sapphire substrate is used. Three big facets, one of which is present at the top center region of the image and the other two of which are located in equivalent positions with $120^{\circ}$ intervals, are also assigned to $\{111\}$ surfaces. Another three large facets whose angular interval is again $120^{\circ}$ are the $\{100\}$ surfaces, and a group of surfaces which are also distributed by $120^{\circ}$ intervals consists of two $\{210\}$ surfaces (this could also be $\{320\}$ at this stage) and a $\{110\}$ in the middle. Although these surfaces are not clearly discernable in fairly small crystallites as shown in Fig. 4 (a) due to the limited resolution of the SEM, a close look of the morphology shown in Fig. 4 (b) confirms that it also has discrete $\{111\},\{100\},\{110\}$, and $\{210\}$ (or $\{320\}$ ) facets.

To determine the uncertainties of the facets developed both in pure and carburized nickel crystals, the Wulffman software [24] was used to simulate the experimentally 
observed morphologies. The focus was made mainly on characterizing the crystallographic identification of the small facets near the top $\{111\}$ surface, since the identity of all the other facets is already clear even from the SEM images due to the inherent symmetric constraints imposed on fcc crystals. By simulating the ECS of crystals with all possible candidates of the surfaces of ambiguity, it is concluded that the surfaces could be either $\{210\}$ or $\{320\}$ depending on the symmetry of the adjacent $\{111\}$ surface. That is, when the symmetry is 6-fold, the surfaces are to be $\{210\}$ as in Fig. 3 (a), while when it is 3 -fold, the surfaces are to be $\{320\}$ as in Fig. 3 (b). Interestingly, however, the facets observed in the carburized nickel crystallites never showed $\{320\}$ surfaces within the resolution limit of SEM, which indicates the $\{320\}$ surfaces observed in the pure nickel system might not be a part of the equilibrium shape but kinetically existing metastable ones. However, this hypothesis needs more systematic and carefully controlled experiments to be confirmed.

The software-generated ECS's of pure nickel for Fig. 3 (a) and (b) and that of the carburized nickel for Fig. 4 (a) or (b) are presented in Fig. 5 (a), (b), and (c), respectively. It should be noted that in Fig. 5 (a) the areal fraction of $\{210\}$ is even larger than or at least comparable to that of $\{111\}$ that is commonly known as the most stable surfaces in fcc with the lowest energy, but is almost negligibly small in Fig. 5 (b). If we assume that the observed shape in Fig. 3 (a) is the true ECS of the pure nickel, the relative energies of each surface normalized by the energy of (111) surface 
are given as $\gamma_{100} / \gamma_{111}=1.01, \gamma_{210} / \gamma_{111}=0.95$, and $\gamma_{110} / \gamma_{111}=1.0$. This makes the rank of the surface energies in the pure nickel $\gamma_{210}<\gamma_{110}<\gamma_{111}<\gamma_{100} \square \gamma_{320}$, which is considerably different from that of other fcc metals. On the other hand, if we assume that the shape in Fig. 3 (b) is the true ECS, the relative surface energies normalized become $\gamma_{100} / \gamma_{111}=1.11, \quad \gamma_{110} / \gamma_{111}=1.02, \quad \gamma_{210} / \gamma_{111}=1.06$, and $\gamma_{320} / \gamma_{111}=1.01$, which makes the rank $\gamma_{111}<\gamma_{320}<\gamma_{110}<\gamma_{210}<\gamma_{100}$, which is again quite different from that of other fcc metals. However, if we only focus on the three principal surfaces, i.e. $\{111\},\{100\}$, and $\{110\}$, the rank of the energy of each surface is in a good agreement with that determined by first principles calculations [25].

The broken bond model, which is commonly used to roughly estimate the surface energies of simple metallic elements, states that the ECS of metals with the fcc symmetry consists of three major low-index surfaces, i.e. $\{111\},\{100\}$, and $\{110\}$. Although it is frequently reported that the ECS predicted by this simple approach has a certain degree of deviations from that observed experimentally, no known experimental work that has a significant deviation from the ones that this approach predicts has been reported yet. For example, pure copper [26] and pure germanium [27] were reported to have additional surfaces in their ECS's, which were proposed as $\{311\}$ facets whose energy is still considerably high in terms of the broken bond model. Though the unexpected surfaces were observed, the rank of the energies of each surface was always in conformity with that predicted by the model. In this regard, 
the abnormality in the rank of the pure nickel currently observed may be attributed to the fact that none of the crystallites could reach its true equilibrium state due to a significant energy barrier for a removal and formation of facet surfaces [13, 20]. This is also strongly supported by the fact that the relative surface energies estimated from the carburized nickel crystallites, whose surface energy anisotropy, equivalently step free energy that determines the nucleation energy barrier for the removal and formation of facets is considerably lower, are $\gamma_{100} / \gamma_{111}=1.00, \gamma_{110} / \gamma_{111}=1.04$, and $\gamma_{210} / \gamma_{111}=1.04$, which results in $\gamma_{111}<\gamma_{100}<\gamma_{110} \leq \gamma_{210}$. It is interesting to note that the rank in the surface energies in the carburized nickel is no longer in accordance with that predicted by first principles calculation [25] but agree with that from the broken bond model, except the practically identical energy value of $\{210\}$ with $\{110\}$. This indicates that the lower energy of $\{110\}$ surfaces compared with that of $\{100\}$ surfaces both theoretically expected and experimentally observed in the pure nickel system may be due to a reconstruction effect which is only dominant in the enthalpy-dominant faceted crystals [28]. On the other hand, considering that the kinetics of facet formation is critically susceptible to the changes in the step free energy which is closely related to the surface energy anisotropy (in general, the higher the anisotropy, the slower the kinetics), the observed shape in the carburized nickel system can be safely assumed to be very close to the equilibrium shape. However, the shapes in the pure nickel system should be regarded as kinetically frozen ones near their equilibrium state. In fact, most studies on the equilibrium crystal shapes have 
been done on crystals whose surface energy anisotropy is so small that the shape involves isotropic curved surfaces [17, 26, 29].

So far, the appearance of unexpected surfaces, as in the case of $\{311\}$ in copper [26] and germanium [27], has often been explained only qualitatively in terms of adsorption effect due to the presence of impurities or a surface-modifying atmosphere [30, 31]. However, a decisive theoretical reasoning on the presence of such unexpected surfaces in ECS is still lacking. So is the origin of the developed $\{320\}$ and $\{210\}$ facets in ECS whose energy is almost comparable to that of $\{111\}$ in the current study. One possibility for the dominant appearance of the $\{320\}$ and $\{210\}$ facets is that it might be due to a small amount of remnant oxygen trapped inside the quartz tube during the sealing procedure. As is in conformity with the classical theory, only if the remnant oxygen can be assumed to be preferentially attached only to the surfaces of relatively high Miller indices, this preferential adsorption of oxygen might lead to a disruption in the rank of the surface energies as observed in the current investigation. However, this assumption is not highly plausible, because the oxygen adsorption would take place simultaneously on all the existing surfaces, which means it is not likely that the energy of only a specific surface of a high energy can be the lowered.

Another possibility which seems more relevant to the current case is that these 
surfaces are kinetically-stabilized metastable ones and the unexpected extent of occupancy is merely a consequence of a sluggish kinetics for the removal of these surfaces and the formation of new surfaces of a lower energy [13, 20]. As was extensively reviewed in Ref. [5] and the references therein, the growth of a faceted surface is always followed in a layer-by-layer manner, which requires a two-dimensional nucleation before the formation of any layer is established. The free energy change during the formation of a two-dimensional nucleus on a faceted surface is given as,

$$
\Delta G_{2 D}=-A_{2 D} \cdot \Delta G_{V, 2 D}+\sum_{i} l_{i} \cdot \varepsilon_{i}
$$

where $A_{2 D}$ and $\Delta G_{V, 2 D}$ refer to the area of the nucleus and the driving force for the formation of the nucleus, respectively. Also, $l_{i} \cdot \varepsilon_{i}$ denotes the free energy increase due to the formation of a step, where $l_{i}$ and $\varepsilon_{i}$ are the length of an edge and the corresponding step free energy. From Eq. (1), the critical driving force for the formation of a facet is derived as

$$
\Delta G_{V, 2 D}=\frac{\sum_{i} l_{i} \cdot \varepsilon_{i}}{A_{2 D}},
$$

which indicates that when it is assumed that the same amount of atoms is grouped to form a nucleus, the easiness of the formation of a nucleus mainly depends on the sum of the step-induced excess energies. Since the step free energy is, in definition, equal to the surface energy per an atomic height with the entropic effect ignored, nucleation 
takes place more actively and easily on the surfaces with a higher energy. For example, let us think of an fcc crystal only composed of $\{111\}$ and $\{100\}$ surfaces, as is schematically illustrated in Fig. 6 . The energy ratio between the surfaces was arbitrary taken as $\gamma_{100} / \gamma_{111}=1.076$. If we ignore the entropy contributions to the step free energy, the ratio of the step free energy of $\{100\}$ to that of $\{111\}$ also can be assumed as 1.076. Then, the ratio between the driving forces for the formation of each nucleus is given

$$
\Delta G_{V, 2 D}^{\{100\}} / \Delta G_{V, 2 D}^{\{111\}}=0.815
$$

which implies that the surfaces with a higher energy is energetically easier to be formed. This also indicates that the surfaces with a higher energy would eventually grow out of existence, being replaced by the surfaces with a lower energy, as long as the atomic flux is high enough to guarantee the driving force for the nucleation. However, when a morphological change of a crystal is highly dependent on a surface diffusion due to a limited supply of the atomic flux, the driving force is mostly not enough to remove those energetically unstable surfaces, because even the removal of a faceted surface has an energy barrier [13, 20].

Figure 7 shows an XPS spectrum of the $2 p$ region for the ECS of the pure as well as carburized nickel. It can be noted that the binding energy peak of the carburized nickel in Fig. 7 shows a slight negative shift. It is known that the presence of binding states associated with the adsorbed atoms of a high electronegativity such as carbon, oxygen, 
and etc. induces a positive shift in XPS spectra [32]. Therefore, the drastic shift induced by carbon may not be attributed to the adsorption of carbon on the surface but a solid solution of carbon into the nickel lattice, because the interstitial type of solid solution as in the case of the current study is known to decrease the binding energy of the system, resulting in a negative shift in the XPS spectra [33-35]. In fact, a number of reports in the literature have implicitly indicated that a solid solution has a significant influence on the changes in surface energies [36-39]. Especially in the case of systems involving an interstitial solid solution, the surface energy decreases as the level of solid solution increases [38]. Because the decrease in the surface energy is mainly due to an entropic effect induced by the incorporation of foreign atoms into the lattice interstitial sites, this type of interstitial solid solution is expected to result in a decrease in the directionality in binding energy, and a consequent reduction in the surface energy anisotropy. Therefore, the changes in the ECS of nickel by the introduction of carbon could be attributed to an effect of the solid solution between nickel and carbon, the possibility of which has not been considered theoretically in the literature yet. 


\section{Conclusion}

The equilibrium shape of the pure and carburized nickel systems was investigated. Due to a sluggish kinetics, only a near equilibrium shape consisting of $\{111\},\{100\}$, $\{110\},\{210\}$, and $\{320\}$ surfaces was observed in the pure nickel crystallites. Those kinetically stabilized shapes suggested that not only the formation but also the removal of faceted surfaces have a significant energy barrier, which is in a good conformity with the theoretical prediction [13]. On the other hand, it was observed that the presence of carbon results in a drastic decrease in the surface energy anisotropy, which was evident from the changes in the shape from a polyhedral with sharp edges to a sphere with some isolated facets. The role of carbon in reducing the surface energy anisotropy was explained in terms of the changes in the binding state features of nickel crystals due to the solid solution of carbon into a nickel lattice. 
This work has been financially supported by the Ministry of Education \& Human Resources Development of the Korean Government through the Brain Korea 21 Project. 


\section{References}

[1] W.H. Murphy and W.S. Walston, US Patent 5695821 (1997).

[2] D.F.K. Hennings, J. Eur. Ceram. Soc. 21 (2001) p.1637.

[3] V. Randle and D. Horton, Scripta Metall. Mater. 31 (1994) p.891.

[4] S.B. Lee, N.M. Hwang, D.Y. Yoon and M.F. Henry, Metall. Mater. Trans. A 31 (2000) p.985

[5] W. Jo , D.Y. Kim and N.M. Hwang, J. Am. Ceram. Soc. 89[8] (2006) p.2369.

[6] R.D. Doherty, Met. Sci. 16 (1982) p.1.

[7] P.A. Manohar, M. Ferry and T. Chandra, ISIJ Internatonal 38 (1998) p.913.

[8] T. Laser, Ch. Hartig, M.R. Nürnberg, D. Letzig and R. Bormann, Acta Mater. 56 (2008) p.2791

[9] G.S. Rohrer, Annu. Rev. Mater. Res. 35 (2005) p.99.

[10] N.M. Hwang, D.K. Lee, K.J. Ko, B.J. Lee, J.T. Park, B.D. Hong, J.K. Kim and D.Y. Kim, Solid-to-Solid Phase Transform. Inorganic Mater. 1 (2005) p.591

[11] S.J.L. Kang, Sintering: Densification, Grain growth and Microstructure, Elsevier, Amsterdam, 2005

[12] T.B. Massalski, H. Okamoto, P.R. Subramanian and L. Kacprzak, Binary alloy phase diagrams, Materials Park, ASM international, Ohio, 1 (1990) p.866.

[13] W.W. Mullins and G.S. Rohrer, J. Am. Ceram. Soc. 83[1] (2000) p.214.

[14] M. Kitayama, T. Narushima and A.M. Glaeser, J. Am. Ceram. Soc. 83[10] (2000) 
p.2572.

[15] H. Gabrisch, L. Kjeldgaard, E. Johnson and U. Dahmen, Acta Mater. 49 (2001) p.4259.

[16] B.E. Sundquist, Acta Metall. 12 (1964) p.67.

[17] J.C. Heyraud and J.J. Metois, J. Crystal Growth 50 (1980) p.571.

[18] J.H. Choi and D.Y. Kim, J. Am. Ceram. Soc. 80 (1997) p.62.

[19] J.H. Choi, D.Y. Kim, B.J. Hockey, S.M. Wiederhorn, J.E. Blendell and C.A. Handwerker, J. Am. Ceram. Soc. 85 (2002) p.1841.

[20] M. Degawa and E.D. Williams, Surf. Sci. 595 (2005) p.87.

[21] M. Humenik (JR) and W.D. Kingery, J. Am. Ceram. Soc. 37 (1954) p.18.

[22] G. Levi, C. Scheu and W. Kaplan, Inter. Sci. 9 (2001) p.213.

[23] R.M. Crispin and M. Nicholas, J. Mater. Sci. 11 (1976) p.17.

[24] A.R. Roosen, R.P. Mc Cormack and W.C. Carter, Comput. Mater. Sci. 11(1) (1998) p.16. Available at http://www.ctcms.nist.gov/wulffman/

[25] L. Vitos, A.V. Ruban, H.L. Skriver and J. Kollár, Surf. Sci. 411 (1998) p. 186.

[26] D. Chatain, V. Ghetta and P. Wynblatt, Interface Sci. 12 (2004) p.7.

[27] D.J. Eaglesham, F.C. Unterwald and D.C. Jacobson, Phys. Rev. Lett. 70 (1993) p.966.

[28] J.M. Howe, Interfaces in Materials, John Wiley \& Sons, New York, 1997, P. 94. [29] J.C. Heyraud, J.J. Metois, Surf. Sci. 128 (1983) p.334

[30] A. Pimpinelli and J. Villain, Physics of Crystal Growth, Press, Cambridge 
University, Cambridge, 1998, p.55.

[31] E.D. Williams and N.C. Bartelt, Ultramicroscopy, 31 (1989) p.36.

[32] J.F. Watts and J. Wolstenholme, An Introduction to Surface Analysis by XPS and AES, John Wiley \& Sons Ltd, Chichester, England, 2003, p.65.

[33] T. Hagio, A. Takase and S. Umebayashi, J. Mater. Sci. Lett. 11 (1992) p.878.

[34] R. Inoue, M. Kitagawa, T. Nishigaki, D. Morita, K. Ichino, H. Kusano and H. Kobayashi, Appl. Surf. Sci. 142 (1999) p. 341.

[35] S. Diplas, J.F. Watts, P. Tsakiropoulos, G. Shao, G. Beamson, and J.A.D. Matthew, Surf. Interface Anal. 31 (2001) p.734.

[36] M.C. Inman, D. McLean, H.R. Tipler, Proc. Roy. Soc. A 273 (1963) p.538.

[37] E.D. Hondros, Proc. Roy. Soc. A 286 (1965) p.479.

[38] L.E. Murr, Interfacial phenomena in metals and alloys, Addison-Wesley Pub. Co., Massachusetts, 1975, p.376.

[39] H.O. Mosca and G. Bozzol, Surf. Sci. 601 (2007) p.3224. 


\section{Figure captions}

Fig. 1. SEM micrographs of (a) plane and (b) cross-sectioned view of the deposited nickel thin film.

Fig. 2. SEM micrograph for (a) magnified nickel crystal of cross-sectioned and (b) a bird's eye view of isolated pure nickel crystals, annealed at $1200^{\circ} \mathrm{C}$ for $100 \mathrm{~h}$ under $10^{-4}$ torr atmospheric pressure.

Fig. 3. Near-equilibrium shape of pure nickel crystals, annealed at $1200{ }^{\circ} \mathrm{C}$ for $100 \mathrm{~h}$ under $10^{-4}$ torr atmospheric pressure.

Fig. 4. Near-equilibrium shape of nickel crystals, annealed at $1200{ }^{\circ} \mathrm{C}$ for $100 \mathrm{~h}$ under carburizing atmosphere.

Fig. 5. Software-generated ECS simulated for the experimentally observed pure and carburized nickel crystals shown in Fig. 3 and 4. (a) and (b) correspond respectively to (a) and (b) in Fig. 3, and (c) corresponds to (b) in Fig. 4.

Fig. 6. Schematic of fcc crystal only composed of $\{111\}$ and $\{100\}$ surfaces. The energy ratio between the surfaces was arbitrary taken as $\gamma_{100} / \gamma_{111}=1.076$.

Fig. 7. XPS spectra of nickel $2 p$ region for the (a) pure nickel and (b) carburized crystals. 


1
1
3
4
5
6
7
8
9
10
11
12
13
14
15
16
17
18
19
20
21
22
23
24
25
26
27
28
29
30
31
32
33
34
35
36
37
38
39
40
41
42
43
44
55
50
56
57
48
59
50
51
53
50

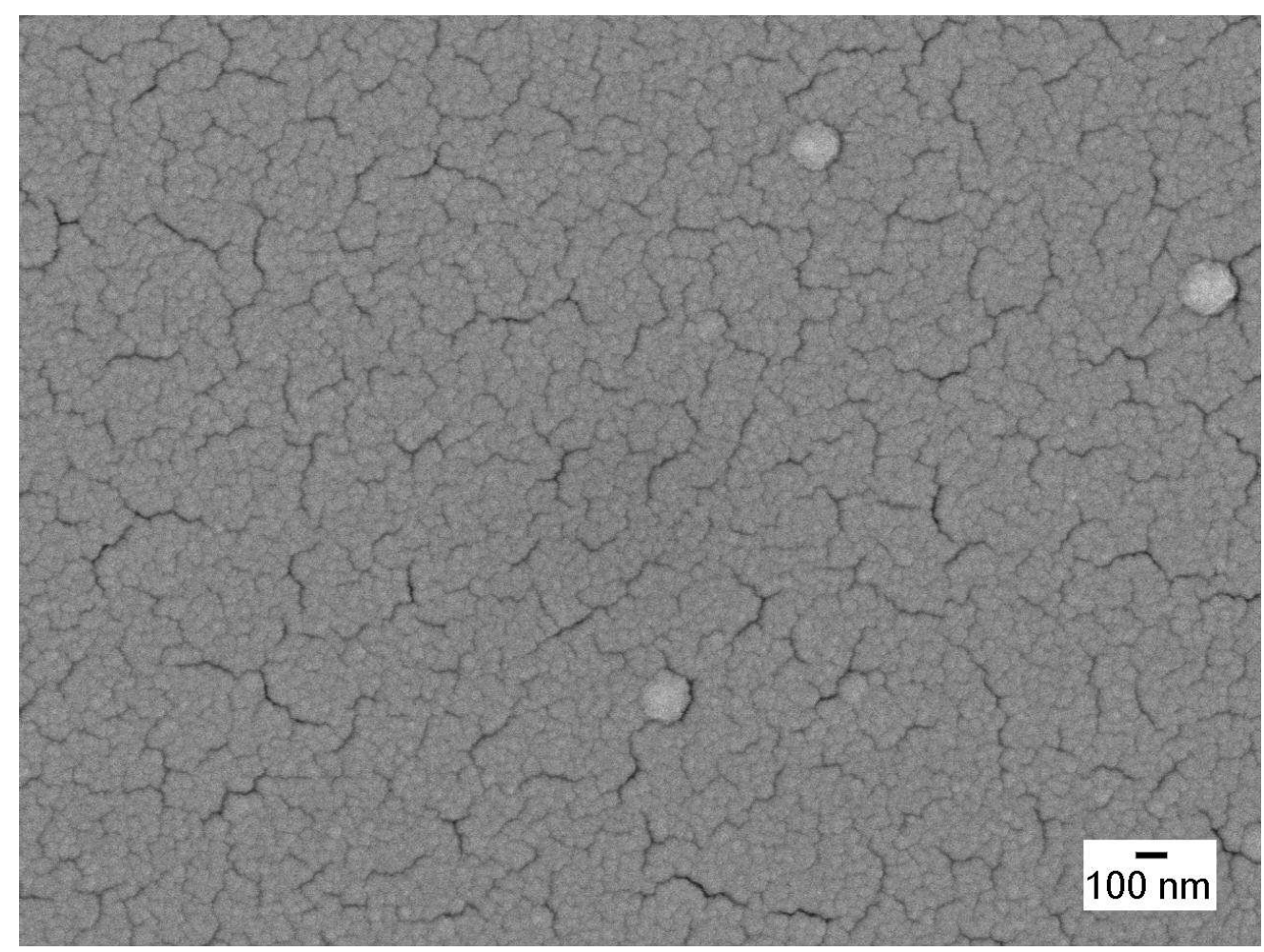

Fig $1 \mathrm{a}$

$338 \times 254 \mathrm{~mm}(96 \times 96 \mathrm{DPI})$ 


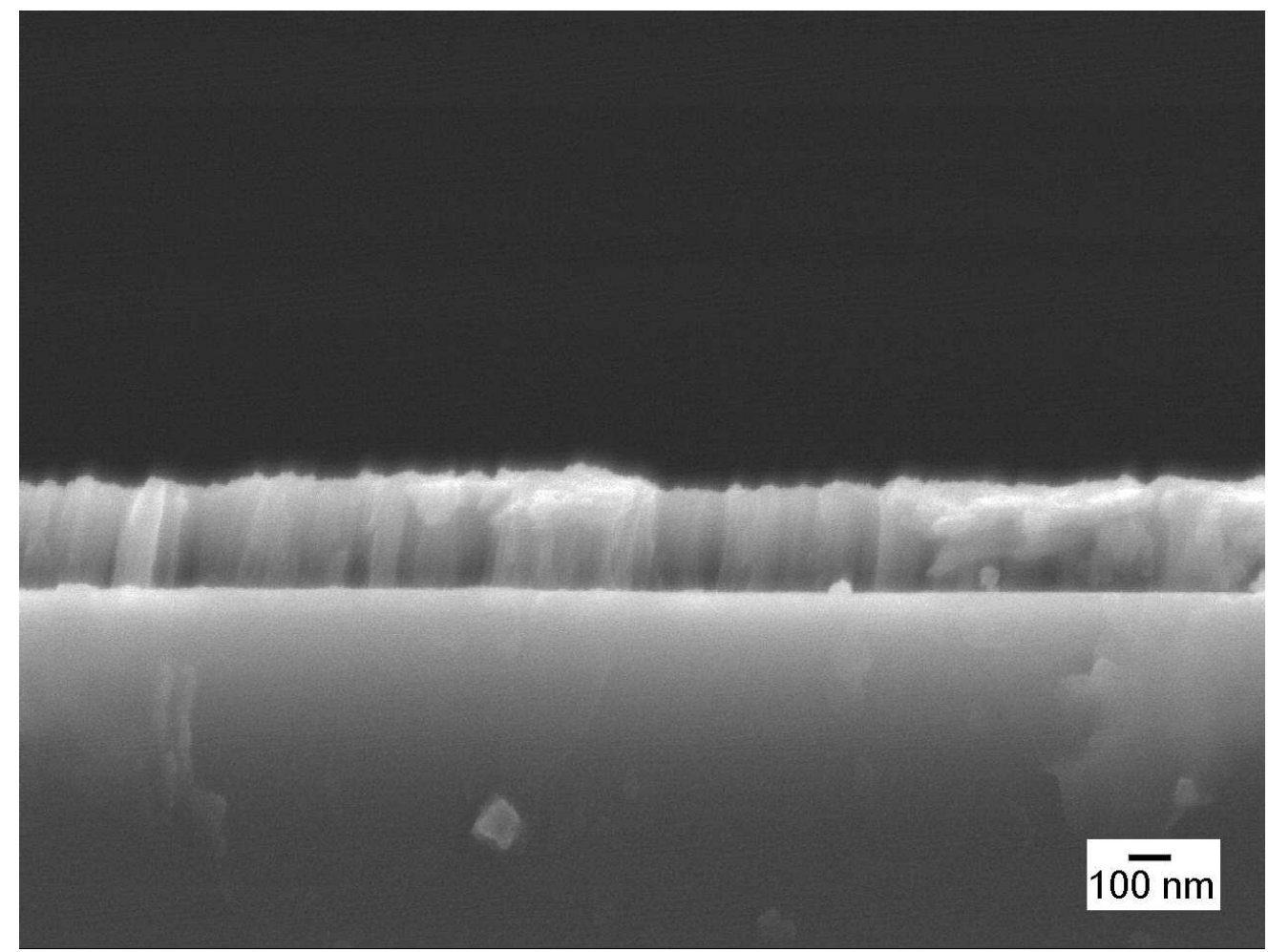

Fig $1 b$

$338 \times 255 \mathrm{~mm}(96 \times 96$ DPI) 


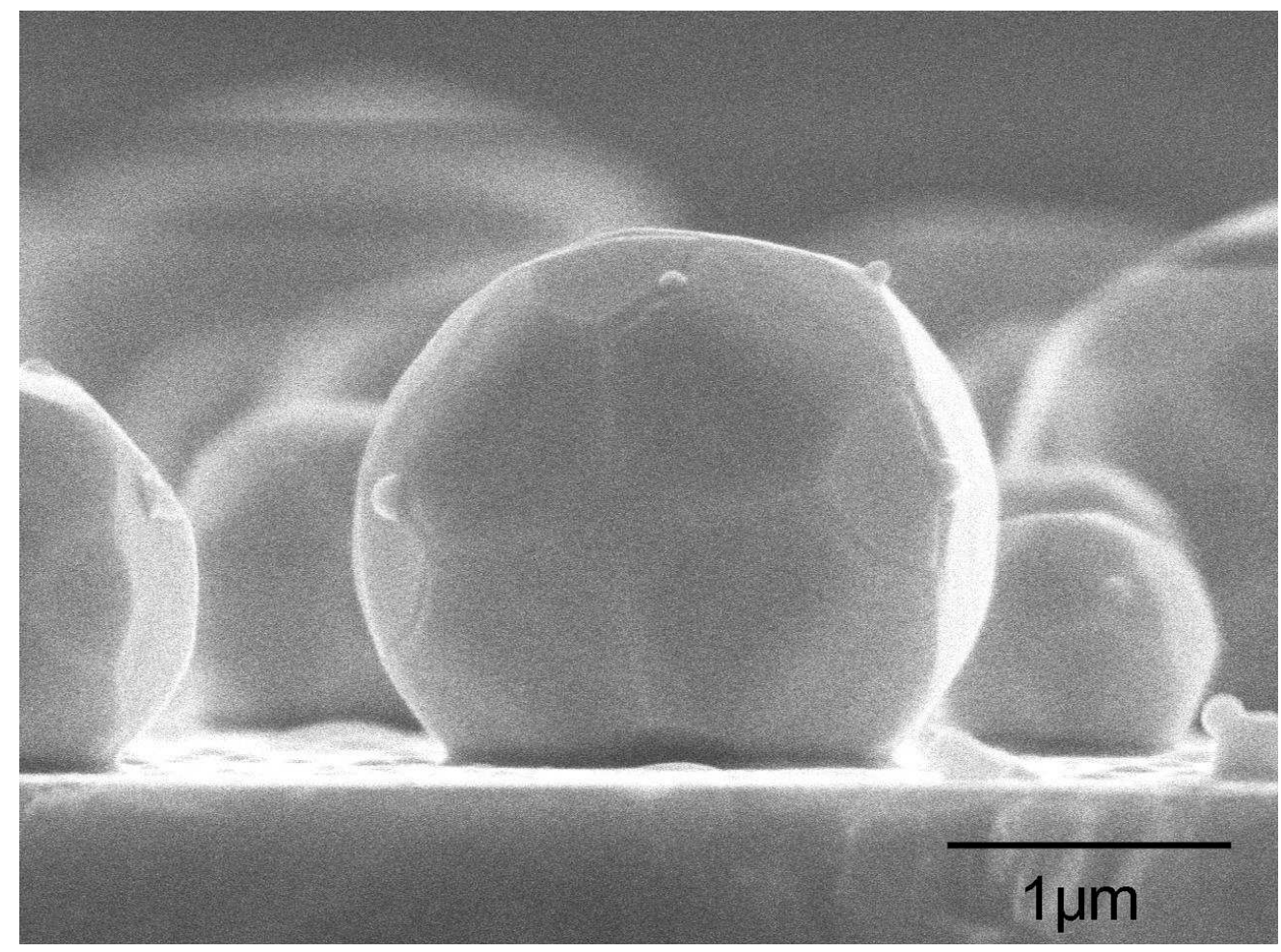

Fig $2 \mathrm{a}$

$338 \times 252 \mathrm{~mm}(96 \times 96 \mathrm{DPI})$ 


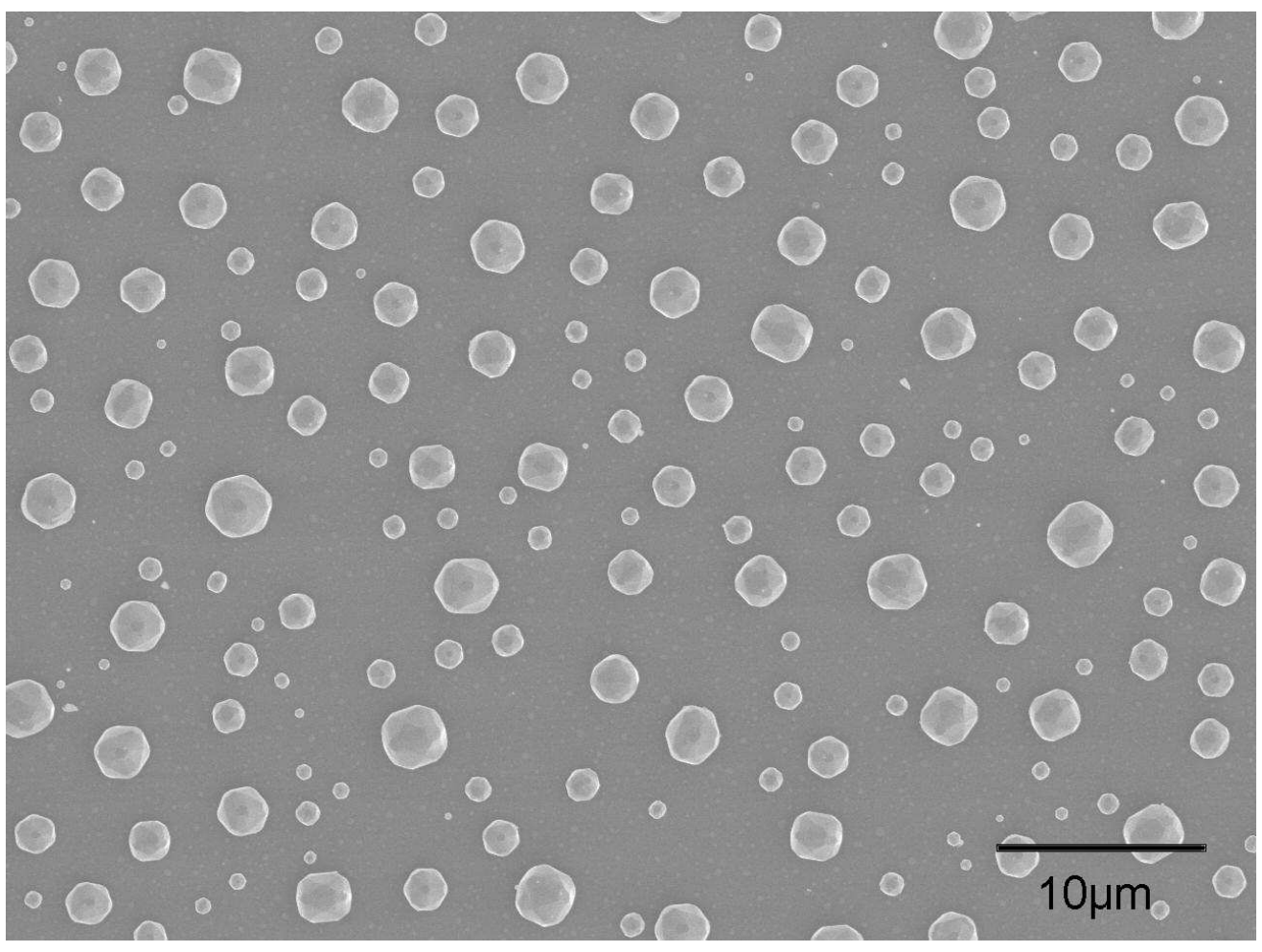

Fig $2 b$

$338 \times 252 \mathrm{~mm}(96 \times 96$ DPI $)$

35

36

37

38

39

40

41

42

43

44

45

46

47

48

49

50

51

52

53

54

55

56 


\section{Page 29 of 35}

Philosophical Magazine \& Philosophical Magazine Letters

1

2

3

4

5

6

7

8

9

10

11

12

13

14

15

16

17

18

19

20

21

22

23

24

25

26

27

28

29

30

31

32

33

34

35

36

37

38

39

40

41

42

43

44

45

46

47

48

49

50

51

52

53

54

55

56

57

58

59

60

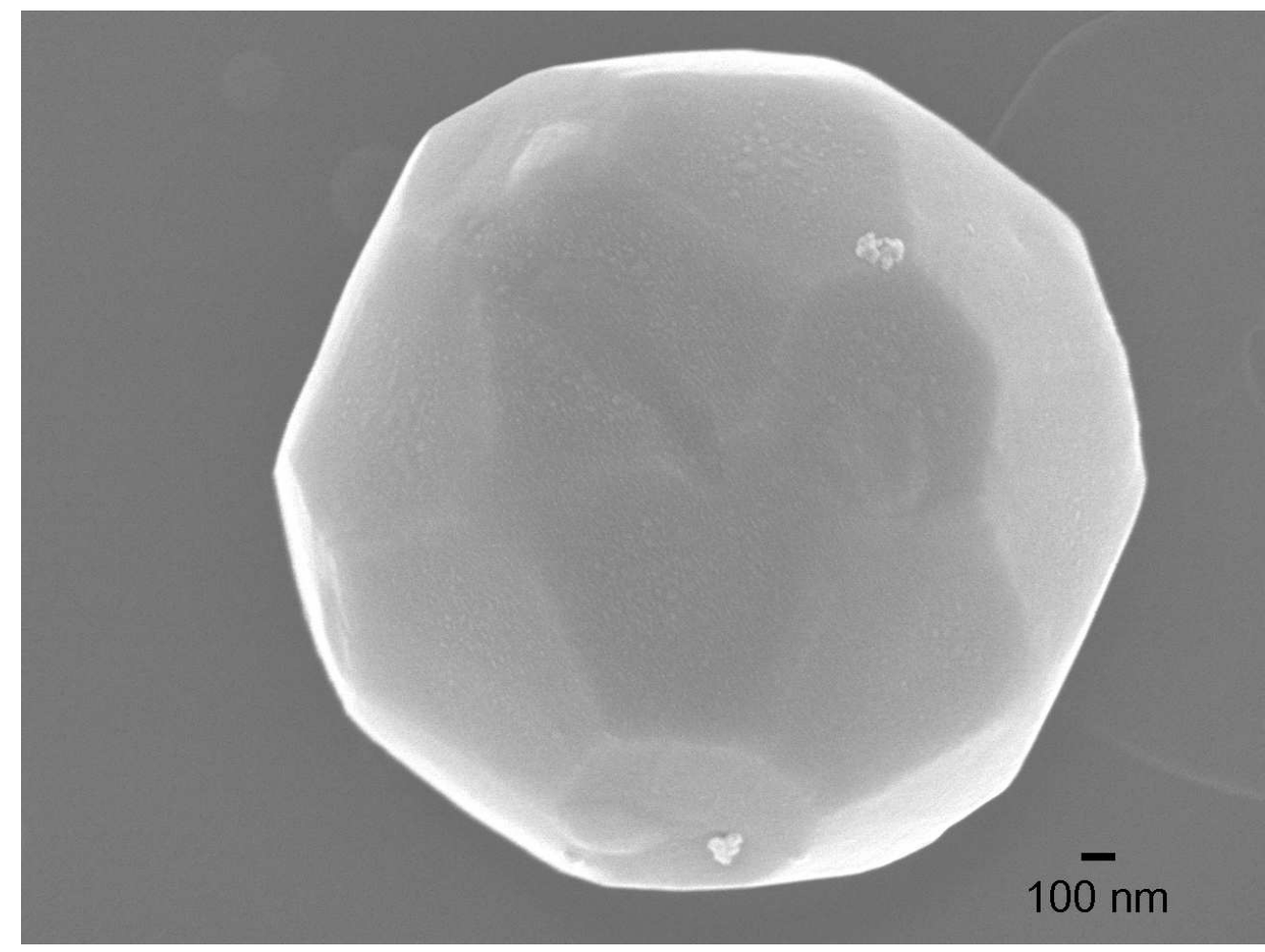

Fig $3 a$

$119 \times 89 \mathrm{~mm}(271 \times 271 \mathrm{DPI})$ 


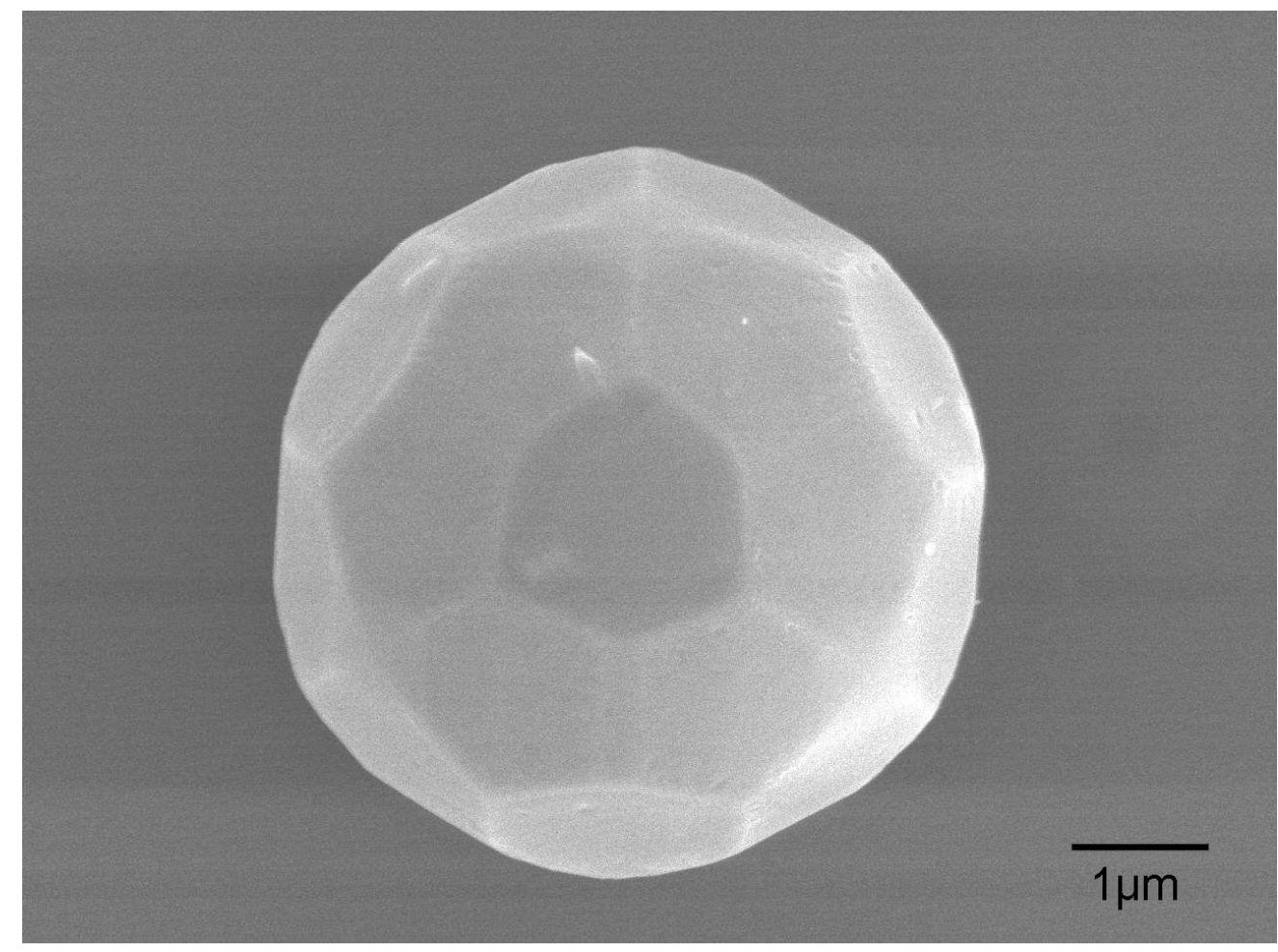

Fig $3 b$

$338 \times 251 \mathrm{~mm}(96 \times 96 \mathrm{DPI})$ 


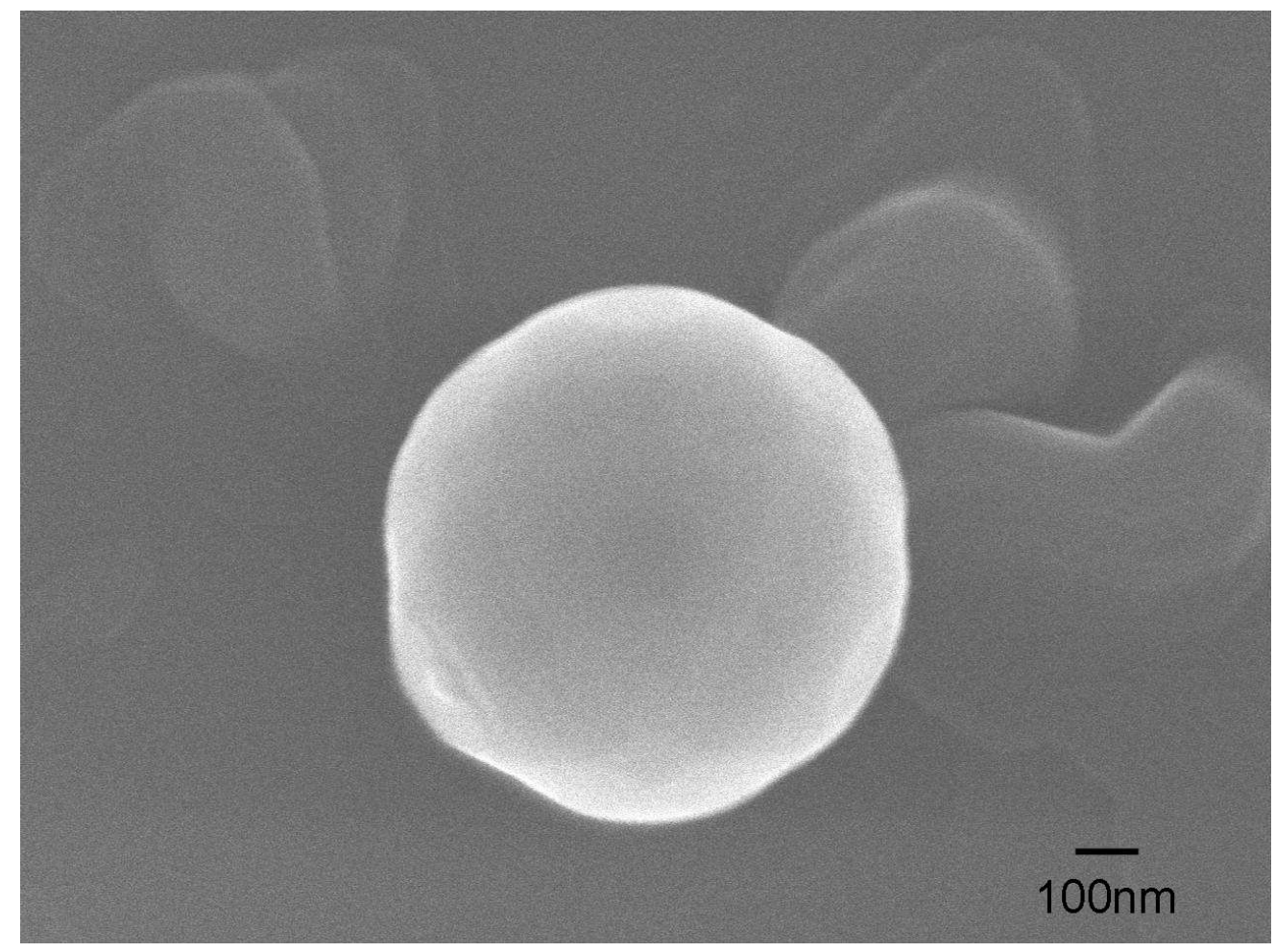

Fig $4 a$

$338 \times 252 \mathrm{~mm}(96 \times 96$ DPI $)$ 


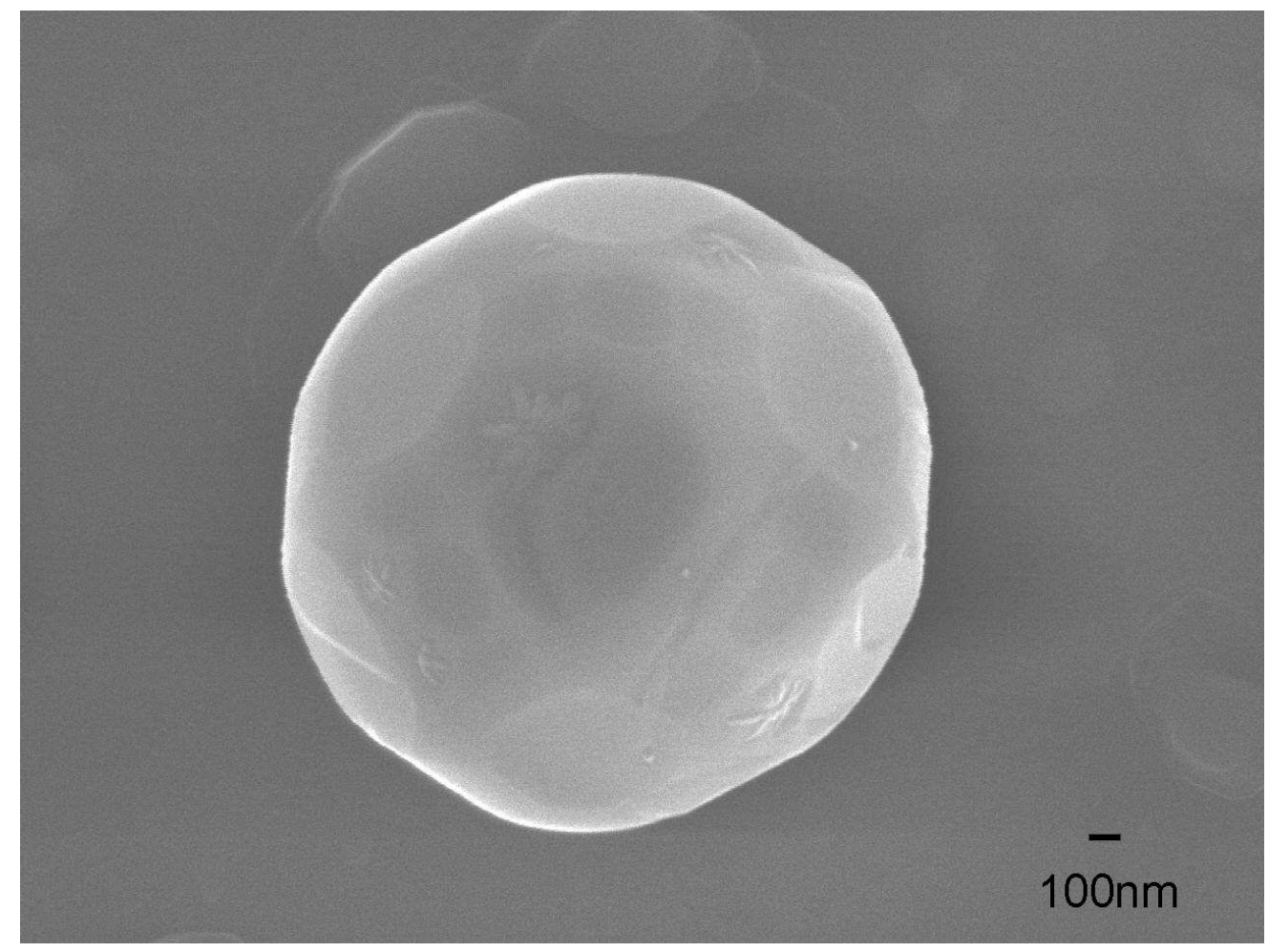

Fig 4b

$338 \times 253 \mathrm{~mm}(96 \times 96$ DPI $)$ 
(a)

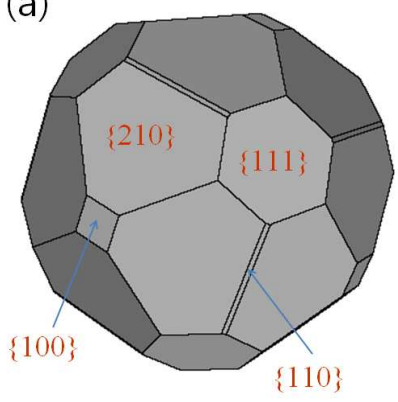

$\{110\}$ (b)

$\{110\}$

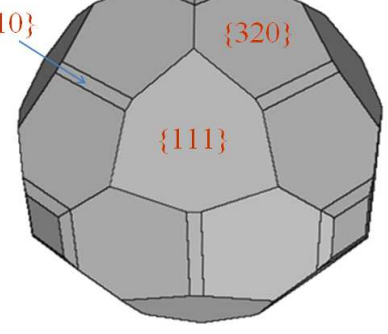

$136 \times 50 \mathrm{~mm}(300 \times 300 \mathrm{DPI})$

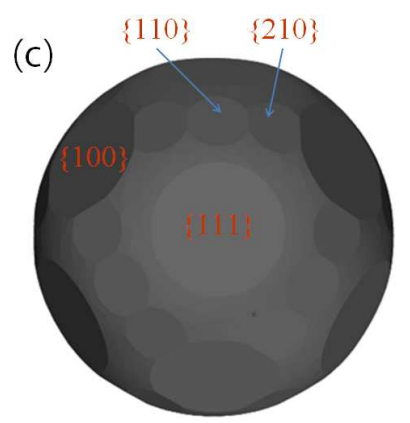

0

22

23

24

26

27

28

29

30

31

33

34

35

36

37

38

39

40

41

42

44

45

46

47

48

49

51

52

53

54

55

56 


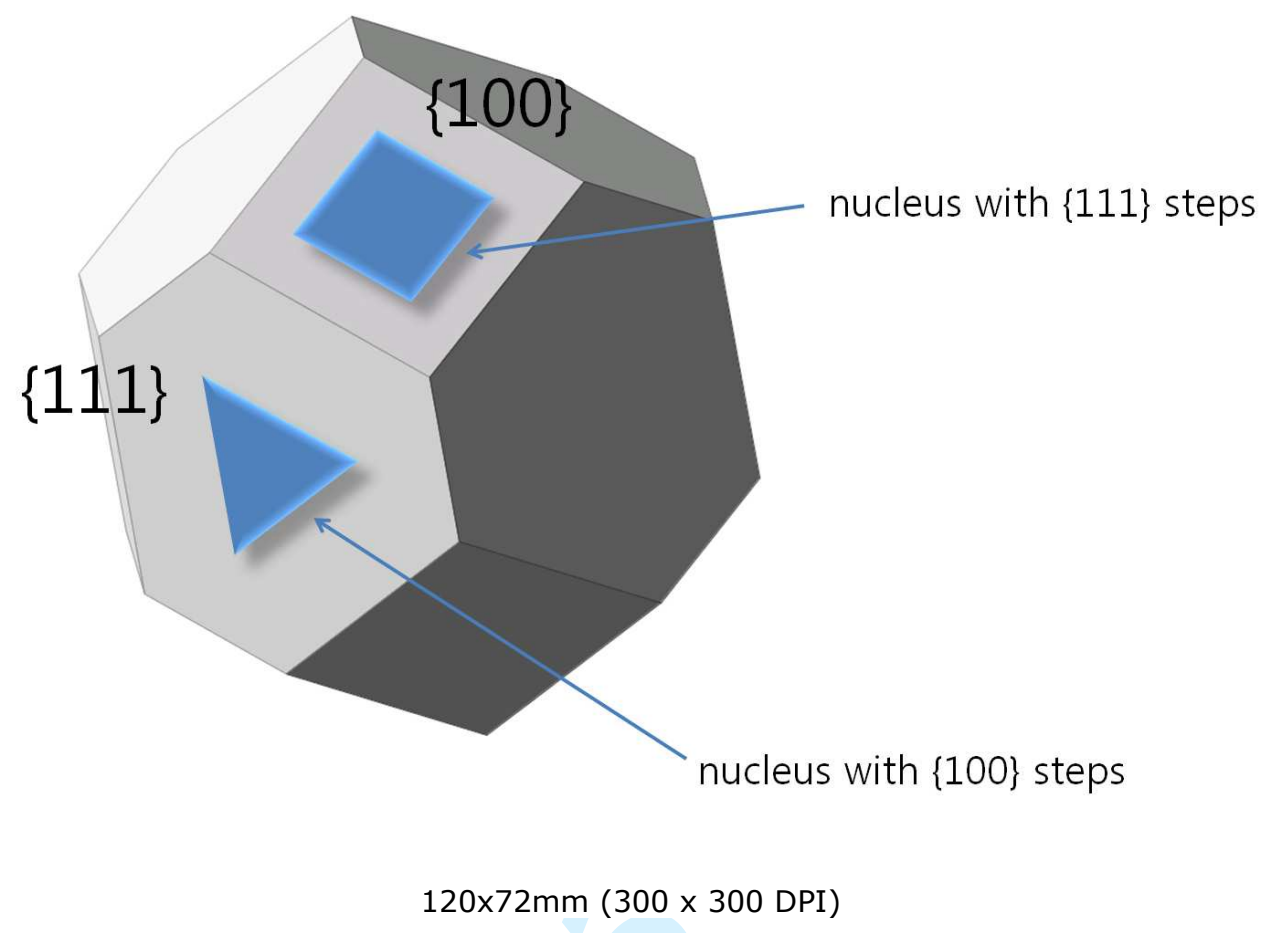




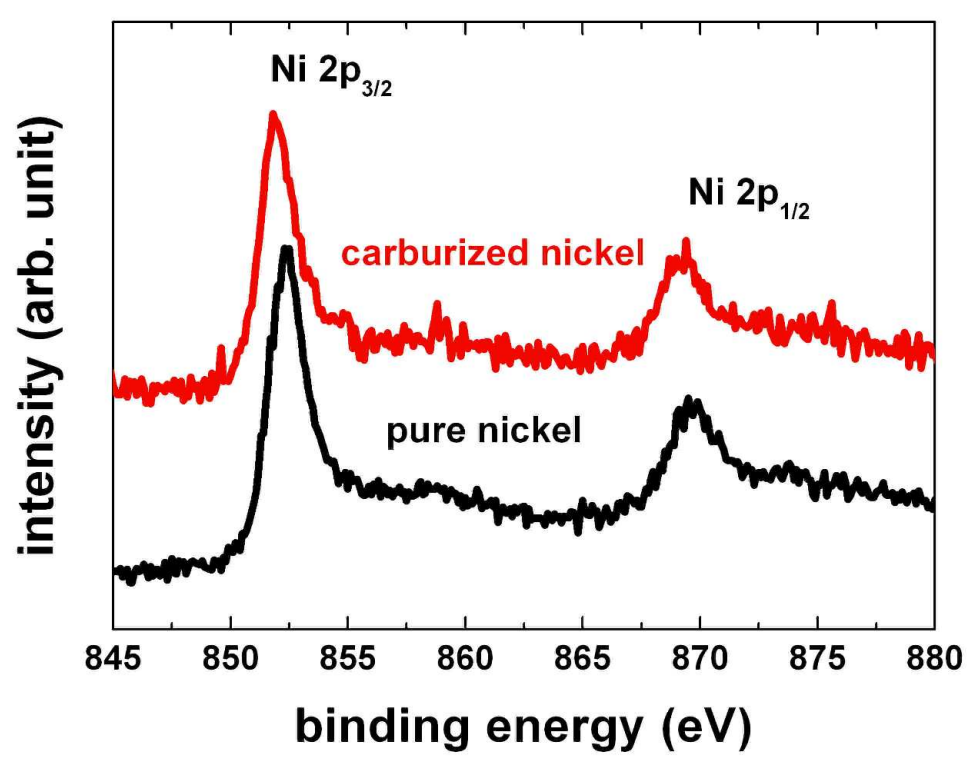

$286 \times 201 \mathrm{~mm}(300 \times 300$ DPI $)$ 